\title{
A case report: paroxysmal nocturnal hemoglobinuria and systemic lupus erythematosus association
}

\author{
Istemi Serin*,1(iD), Aslıhan Bayir² ${ }^{2}$, Hasan Goze ${ }^{1}$ (i) \& Osman Yokus ${ }^{1}$ (iD \\ ${ }^{1}$ Department of Hematology, University of Health Science, Istanbul Training \& Research Hospital, Istanbul, Turkey \\ ${ }^{2}$ Department of Internal Medicine, University of Health Science, Bagcilar Training \& Research Hospital, Istanbul, Turkey \\ *Author for correspondence: Tel.: +90 532315 2393; serinistemi@hotmail.com
}

Paroxysmal nocturnal hemoglobinuria (PNH) is defined by acquired intravascular hemolytic anemia, thrombosis and bone marrow failure with pancytopenia. Systemic lupus erythematosus (SLE) also appears as an autoimmune disease. The coexistence of both is rarely reported. Here we report the case of a 30year-old female presenting with pancytopenia and diagnosed as SLE, who also had a PNH clone. Bone marrow biopsy did not support hypoplastic anemia. The patient was then followed up with the consideration of the existence of a PNH clone with SLE. She was treated by the rheumatology department and complete blood count improved under immunosuppressive treatment. The coexistence of CD59-CD55 deficiency with autoimmune diseases has been reported. It is an important example in terms of receiving clinical response with SLE-specific treatment.

First draft submitted: 28 May 2020; Accepted for publication: 21 September 2020; Published online: 13 November 2020

Keywords: hemolytic anemia $\bullet$ immunosuppressive therapy $\bullet$ pancytopenia $\bullet$ paroxysmal nocturnal hemoglobinuria - systemic lupus erythematosus

Paroxysmal nocturnal hemoglobinuria (PNH) is a clonal hematopoietic stem cell disease manifested by chronic intravascular hemolysis findings, bone marrow failure and thrombosis. It has is an acquired defect of the glycosylphosphatidylinositol associated proteins CD55 and CD59 [1]. These protein deficiencies lead to complementmediated cell lysis and other clinical manifestations. Flow cytometry analysis of antibodies directed against CD55 and CD59 is the gold standard technique for the diagnosis [2-4].

The disease manifests itself in two main clinical pictures: hemolytic and hypoplastic type. Chronic intravascular hemolysis findings are typically more prominent in the hemolytic type, whereas pancytopenia is typically more prominent in the hypoplastic type. Coombs-negative hemolytic anemia (laboratory findings of hemolysis) as well as presence of thrombosis, iron deficiency and/or cytopenia requires a PNH examination. PNH can be found alone (classical PNH) or accompanied by aplastic anemia and myelodysplastic syndrome (MDS) [5]. PNH also has a clinical importance in terms of hematological malignancies with which it can be seen. Presence of PNH clone with MDS and aplastic anemia changes the treatment approach completely. Similarly, because of being a clonal stem cell disease, it also poses risk for the transformation of acute myeloid leukemia. This rate is $1 \%$ within publications [6].

$\mathrm{PNH}$ clone presence with one of subtypes of MDS is not clearly known. In addition, the effect of PNH clone presence on prognosis or leukemic transformation is also controversial. In the series reported by Wang et al., $35 \%$ of patients with refractory anemia had a PNH clone and $20 \%$ of $5 \mathrm{q}$-syndrome patients also developed a PNH clone during the follow-up period. However, deficiency of multiple glucose phosphate isomerase-linked (GPI-linked) proteins in granulocytes was not detected in patients with a diagnosis of sideroblastic anemia, refractory anemia with excessive blast or clear acute myeloid leukemia. In general, it can be stated that acute leukemia transformation is seen less frequently and the prognosis is better for MDS cases with a PNH clone; however, there is no difference at the point of treatment [7]. MDS association with systemic lupus erythematosus (SLE) is extremely rare and is occasionally mentioned in some case reports. This case report shall present a case with PNH clone that was diagnosed with SLE and presented with bicytopenia.

Future Medicine 
(A)

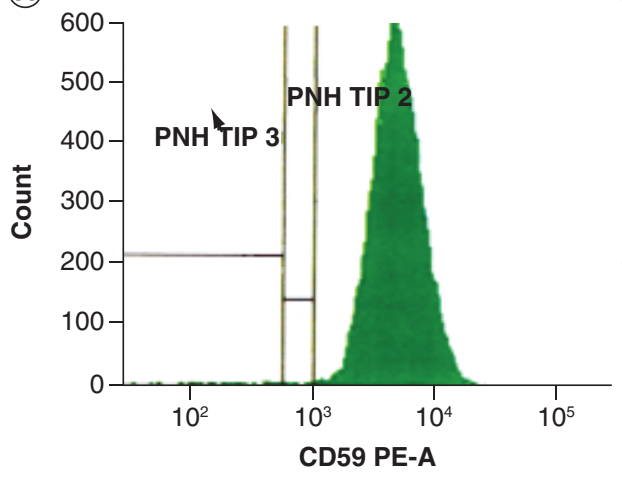

(B)

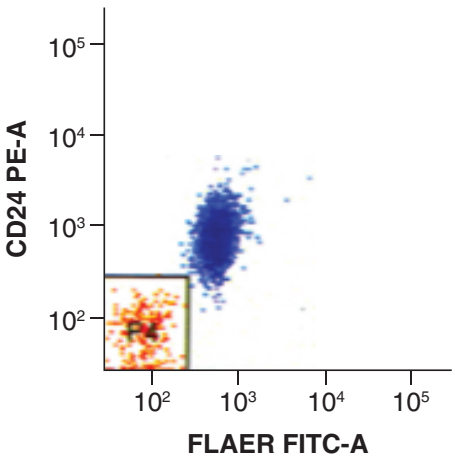

(C)

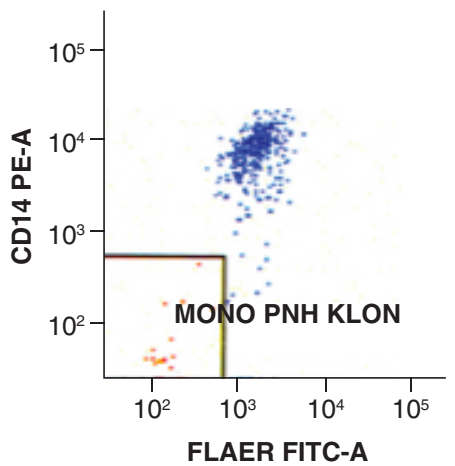

Figure 1. FLAER Test. PNH clones; (A) in erythrocytes, (B) in granulocytes and (C) in monocytes. PNH: Paroxysmal nocturnal hemoglobinuria.

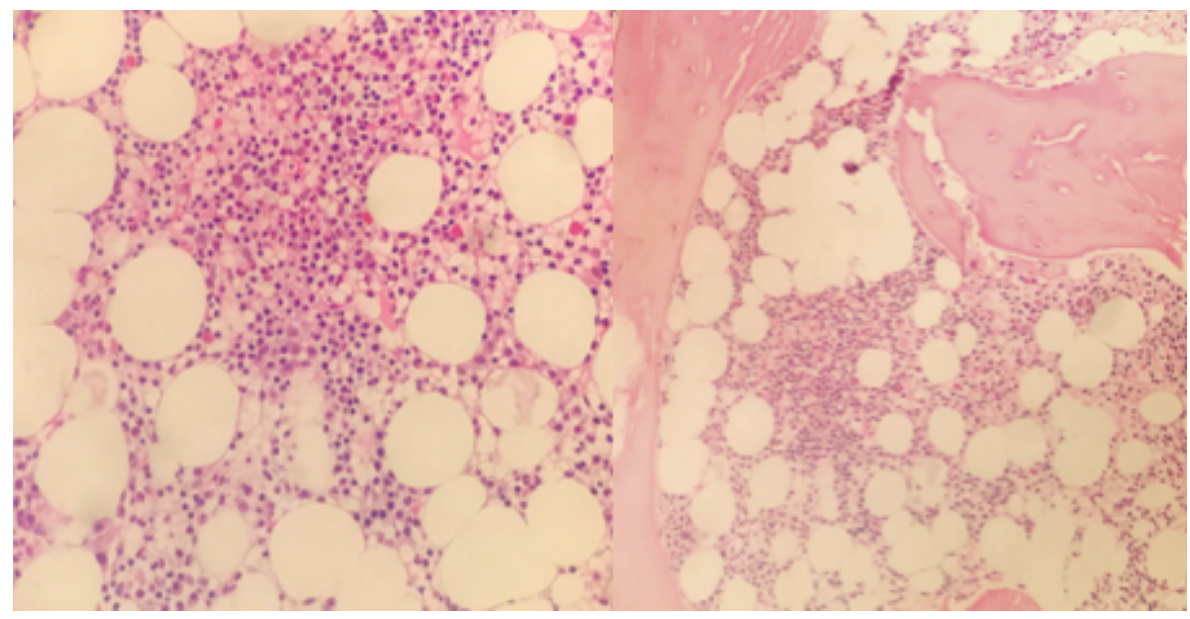

Figure 2. Hematoxylin-eosin staining of the bone marrow specimen.

\section{Case report}

A 30-year-old female patient was examined in the rheumatology clinic for alopecia, bilateral pleural, pericardial effusion and nonerosive oligoarthritis, particularly involving the hand joints. Together with the laboratory results, it fully met the Systemic Lupus International Collaboration Criteria for SLE. She was referred to our hematology clinic on suspicion of aplastic anemia/MDS with a positive FLAER test. She had no complaints other than headache and weakness in her referral. Initial hemoglobin was $5 \mathrm{~g} / \mathrm{dl}$, leukocyte $800 / \mathrm{mm}^{3}$ and platelet was $26,000 / \mathrm{mm}^{3}$. In the biochemical findings, LDH was $400 \mathrm{IU} / 1$ (normal range: 140-280 IU/1). Corrected reticulocyte count was $4.5 \%$, haptoglobin was $0.3(0.5-2.2 \mathrm{~g} / \mathrm{l})$, C3 level was $30 \mathrm{mg} / \mathrm{dl}(80-160 \mathrm{mg} / \mathrm{dl})$ and C4 level was $5 \mathrm{mg} / \mathrm{dl}$ $(10-40 \mathrm{mg} / \mathrm{dl})$. The direct and indirect Coombs tests were negative and the antinuclear antibody titer 1:2560 homogeneous positive, anti-double stranded DNA result was positive. In her peripheral blood smear, it was possible to see reticulocytosis with any other pathological findings. Due to Coombs-negative hemolytic anemia, the PNH clone was rerun with FLAER test and was positive with erythrocytes $0.6 \%, 7.79 \%$ for monocytes and $11.25 \%$ for granulocytes. Figure 1 reveals the presence of PNH clones: $\mathrm{A}$ in erythrocytes, $\mathrm{B}$ in granulocytes and $\mathrm{C}$ in monocytes.

Bone marrow aspiration smears were normocellular with normoblastic erythroid hyperplasia, normal myeloid maturation and adequate megakaryopoesis. There was a mild increase in mature plasma cells, which was considered to be associated with SLE. There was no fibrosis or dysplastic changes in bone marrow specimen (Figure 2).

She was treated in the rheumatology clinic with pulse $1 \mathrm{~g} /$ day for 2 days methylprednisolone treatment. After that, $20 \mathrm{mg}$ of prednisone and cyclosporine $2 \times 50 \mathrm{mg}$ were added to her treatment. Since the need for transfusion completely disappeared by the end of the first month, treatment was continued with the same immunosuppressive therapy. In parallel with the publications that reported that CD59 expression decreased with SLE, the FLAER 
test was repeated and the PNH clone was confirmed. In addition to the stable immunosuppressive treatment, the patient was planned to have rituximab because of refractory oligoarthritis and the patient was taken to the hematology and rheumatology outpatient follow-up.

\section{Discussion}

PNH presents with acquired intravascular hemolytic anemia, large vein thrombosis and pancytopenia clinics with moderate to severe bone marrow failure. In our case, there were no triad findings other than cytopenia. As mentioned, the pathology is based on CD55 and CD59 deficiency. However, CD55 and CD59 deficiency can also be seen in autoimmune thrombocytopenia, hemolytic anemia and systemic lupus erythematosus. It is possible to define them as diseases that create a 'PNH-like phenomenon' [8,9].

Various pathogenetic mechanisms for cytopenia are defined in SLE patients. As an additional factor, the expression of CD55 and CD59 is also reduced in SLE. It has been observed that decreased expression of CD55 and CD59 has a facilitating role in autoimmune hemolytic anemias. The relationship between CD55 expression and antiphospholipid syndrome on erythrocyte surfaces from secondary autoimmune hemolytic anemia to SLE or primary autoimmune hemolytic anemia patients was investigated. In the presence of autoimmune hematolytic anemia, independent from SLE, CD55 and CD59 have been shown to be deficient. However, the relationship between antiphospholipid antibodies and this acquired defect has not yet been established [10,11].

In SLE cases with autoimmune thrombocytopenia and lymphopenia, the role of CD55 and CD59 expression is another topic that has been studied. Lymphopenia in SLE has been associated with autoantibodies that target nuclear antigens. CD55 and CD59 in T and B lymphocytes decreased significantly in SLE patients with lymphopenia. Interestingly, on the contrary, in nonlymphopenic patients with SLE, normal expression was found in $\mathrm{T}$ and $\mathrm{B}$ cells. It has been suggested that deficiency of CD55 and CD59 expression may play a role in the pathophysiology of lymphopenia, possibly by increasing the complement-mediated cytolysis sensitivity of cells. Although there is no correlation between CD55 and CD59 surface density and the presence of any specific autoantibodies, there appears to be a relationship between high anti-double strand DNA, anti-Sm and antiribosomal P antibody titers and lymphopenia [12].

In the presence of PNH clone associated with SLE, there is no data in the literature that clearly demonstrates the pathology of cytopenia. While SLE-related cytopenia can occur with autoantibody-based cell destruction, receptor blockage and apoptosis, the contribution to cytopenia can be mentioned in the presence of PNH clone. Considering SLE cases in which decreased expression of CD55 and CD59 is associated with hemolytic anemia and lymphopenia, it can be said that in our case, the presence of PNH clone contributed to complement-related cell destruction [13].

We present a case about this rare association. Instead of PNH specific therapy, the treatment of the underlying disease makes a clinically significant difference. Treatment was started by evaluating our patient's current clinical picture secondary to SLE, not the PNH clone. Therefore, determining the cause of the clinical picture becomes important in the association of rare diseases. In our case, corticosteroid was started first and as maintenance, it was planned to continue with cyclosporin. Although cytopenia seems to improve with the current treatment, rituximab was added to the treatment due to the continuing joint complaints of the patient and rheumatological follow-up continues under this plan. In the presence of resistant cytopenia, the addition of PNH-specific therapy may come to mind. In the treatment of $\mathrm{PNH}$, the effect of corticosteroids decreasing hemolysis, even if not in every patient, can be observed. Corticosteroid therapy can be effective in increasing hemoglobin levels, especially during periods of refractory hemolysis. Side-effects in long-term treatment limit their use. With targeting complement C5 protein, eculizumab is a treatment option that has been shown to significantly reduce hemolysis and thus hemolysis-related side-effects in PNH cases. In this context, in the presence of resistant cytopenia, specific treatment for the PNH clone may be the subject of controversy in the future.

\section{Conclusion}

The coexistence of CD59-CD55 deficiency with autoimmune hemolytic anemia, autoimmune thrombocytopenia or SLE have been reported. Therefore, the factor causing PNH clone in our case is associated with SLE and it is an important example for clinicians in terms of receiving a clinical response with SLE-specific treatment. 
Executive summary

- Paroxysmal nocturnal hemoglobinuria (PNH) is a clonal hematopoietic stem cell disease manifested by chronic intravascular hemolysis findings, bone marrow failure and thrombosis.

- PNH has a clinical importance in terms of hematological malignancies with which it can be seen. Because of being a clonal stem cell disease, it also poses risk for the transformation of acute myeloid leukemia.

- Its association with systemic lupus erythematosus is extremely rare and is occasionally mentioned in some case reports.

- However, CD55 and CD59 deficiency can also be seen in autoimmune thrombocytopenia, hemolytic anemia and systemic lupus erythematosus (SLE); it is possible to define them as diseases that create a 'PNH-like phenomenon'.

- CD55 and CD59 in T and B lymphocytes are decreased significantly in SLE patients with lymphopenia.

- It has been suggested that deficiency of CD55 and CD59 expression may play a role in the pathophysiology of lymphopenia, possibly by increasing the complement-mediated cytolysis sensitivity of cells.

- Considering SLE cases in which decreased expression of CD55 and CD59 is associated with hemolytic anemia and lymphopenia, it can be said that the presence of a PNH clone contributed to complement-related cell destruction.

- In the treatment of PNH, the effect of corticosteroids decreasing hemolysis, even if not in every patient, can be observed.

- Corticosteroid therapy can be effective in increasing hemoglobin levels, especially during periods of refractory hemolysis.

- By targeting complement C5 protein, eculizumab is a treatment option that has been shown to significantly reduce hemolysis and thus hemolysis-related side-effects in PNH cases. In the presence of resistant cytopenia, specific treatment for the PNH clone may be the subject of controversy in the future.

\section{Acknowledgments}

We respectfully remember all the colleagues we lost in the COVID-19 fight.

Financial \& competing interests disclosure

The authors have no relevant affiliations or financial involvement with any organization or entity with a financial interest in or financial conflict with the subject matter or materials discussed in the manuscript. This includes employment, consultancies, honoraria, stock ownership or options, expert testimony, grants or patents received or pending, or royalties.

No writing assistance was utilized in the production of this manuscript.

Ethical conduct of research

A written informed consent was obtained from our patient to share and publish this case presentation.

Open access

This work is licensed under the Attribution-NonCommercial-NoDerivatives 4.0 Unported License. To view a copy of this license, visit http://creativecommons.org/licenses/by-nc-nd/4.0/

\section{References}

Papers of special note have been highlighted as: $\bullet$ of interest; $\bullet \bullet$ of considerable interest

1. Ruiz-Delgado GJ, Vázquez-Garza E, Méndez-Ramírez N, Gómez-Almaguer D. Abnormalities in the expression of CD55 and CD59 surface molecules on peripheral blood cells are not specific to paroxysmal nocturnal hemoglobinuria. Hematology 14(1), 33-37 (2009).

2. Zacks MA, Garg N. Recent developments in the molecular, biochemical and functional characterization of GPI8 and the GPI-anchoring mechanism [review]. Mol. Membr. Biol. 23(3), 209-225 (2009).

3. Rocha JMC, Silva ML, Souza MEdeL, Murao M, Araújo SSdaS, Santos SME. Detection of PNH cells by flow cytometry, using multiparameter analysis. J. Bras. Patol. Med. Lab. 50(2), 105-114 (2014).

4. Manivannan P, Ahuja A, Pati HP. Diagnosis of paroxysmal nocturnal hemoglobinuria: recent advances. Indian J. Hematol. Blood Transfus. 33(4), 453-462 (2017).

5. Illingworth A, Marinov I, Sutherland DR, Wagner-Ballon O, DelVecchio L. ICCS/ESCCA consensus guidelines to detect GPI-deficient cells in paroxysmal nocturnal hemoglobinuria (PNH) and related disorders part 3 - data analysis, reporting and case studies. Cytometry $B$ Clin. Cytom. 94(1), 49-66 (2018).

- Guidelines state that paroxysmal nocturnal hemoglobinuria can be found alone (classical paroxysmal nocturnal hemoglobinuria) or accompanied by aplastic anemia and myelodysplastic syndrome.

6. Ruiz-Argüelles A, Llorente L. The role of complement regulatory proteins (CD55 and CD59) in the pathogenesis of autoimmune hemocytopenias. Autoimmun. Rev. 6(3), 155-161 (2007). 
- Presence of paroxysmal nocturnal hemoglobinuria clone with myelodysplastic syndrome and aplastic anemia changes the treatment approach completely. Similarly, because of being a clonal stem cell disease, it also poses risk for the transformation of acute myeloid leukemia. This rate is $1 \%$ within publications.

7. Young NS. Paroxysmal nocturnal hemoglobinuria and myelodysplastic syndromes: clonal expansion of PIG-A-mutant hematopoietic cells in bone marrow failure. Haematologica 94(1), 3-7 (2009).

-. In general, it can be stated that acute leukemia transformation is less seen and the prognosis is better in myelodysplastic syndrome cases with a paroxysmal nocturnal hemoglobinuria clone; however, there is no difference at the point of treatment.

8. Nakamura N, Sugawara T, Shirato K et al. Paroxysmal nocturnal hemoglobinuria in systemic lupus erythematosus: a case report. J. Med. Case Rep. 5, 550 (2011).

- CD55 and CD59 deficiency can also be seen in autoimmune thrombocytopenia, hemolytic anemia and systemic lupus erythematosus. It is possible to define them as diseases that create a 'paroxysmal nocturnal hemoglobinuria-like phenomenon'.

9. Luzzatto L, Gianfaldoni G. Recent advances in biological and clinical aspects of paroxysmal nocturnal hemoglobinuria. Int. J. Hematol. 84(2), 104-112 (2006).

10. Maciejewski JP, Rivera C, Kook H, Dunn D, Young NS. Relationship between bone marrow failure syndromes and the presence of glycophosphatidyl inositol-anchored protein-deficient clones. Br. J. Haematol. 115(4), 1015-1022 (2001).

11. Gupta A, Al Fulaij R, Gupta RK, Gupta G, Marouf R. Development of paroxysmal nocturnal haemoglobinuria in systemic lupus erythematosus: an unusual cause of portal vein thrombosis. Lupus 18(8), 743-674 (2009).

12. Ruiz-Argüelles A, Llorente L. The role of complement regulatory proteins (CD55 and CD59) in the pathogenesis of autoimmune hemocytopenias. Autoimmun Rev. 6(3), 155-161 (2007).

- There is no correlation between CD55 and CD59 surface density and the presence of any specific autoantibodies, there appears to be a relationship between high antidouble stranded DNA, anti-Sm and antiribosomal $\mathrm{P}$ antibody titers and lymphopenia.

13. García-Valladares I, Atisha-Fregoso Y, Richaud-Patin Y et al. Diminished expression of complement regulatory proteins (CD55 and CD59) in lymphocytes from systemic lupus erythematosus patients with lymphopenia. Lupus 15(9), 600-605 (2006). 